\title{
O uso de diferentes valores de tarifa como estratégia de transferência de demanda em sistemas de transporte público urbano
}

\author{
The use of different fare values as a demand transfer strategy in urban public \\ transportation systems
}

Ligia Rabay [a] (1), Nilton Pereira de Andrade [a] (1)

[a] Universidade Federal da Paraíba (UFPB), João Pessoa, PB, Brasil.

Como citar: Rabay, L., \& Andrade, N. P. (2019). O uso de diferentes valores de tarifa como estratégia de transferência de demanda em sistemas de transporte público urbano. urbe. Revista Brasileira de Gestão Urbana, 11, e20180024. https://doi.org/10.1590/2175-3369.011.001.A007

\section{Resumo}

Um dos maiores problemas das cidades de médio e grande porte nos dias de hoje é a mobilidade urbana, cujas soluções passam necessariamente pelos sistemas de transporte público coletivo, os quais exigem elevados investimentos em infraestrutura. A frota desse serviço é determinada com base na quantidade de passageiros a serem transportados nos períodos de pico e tem importante papel na definição do valor da tarifa. A diferenciação do valor da tarifa por faixa horária ao longo do dia, embora ainda muito incipiente no Brasil, é uma estratégia bastante utilizada no exterior para diminuir a demanda nos períodos de pico. A oferta de descontos no valor da tarifa para os horários de menor demanda pode atrair parte dos usuários que se desloca nos picos. Dessa forma, o artigo apresenta um minucioso estudo sobre o tema, com uma análise de viabilidade do uso da tarifa diferenciada por horário no sistema de transporte público de uma cidade brasileira. Os resultados mostraram que 69\% dos usuários do sistema no período de pico estariam dispostos a mudar o horário da viagem para obter alguma economia, que o percentual de adesão varia em função da dimensão do desconto oferecido e que existe um desconto mínimo a partir do qual a mudança ocorreria. A estratégia se mostrou viável com boas chances de modificar o perfil da variação da demanda ao longo do dia e contribuir para a redução da frota e dos custos operacionais do sistema.

Palavras-chave: Precificação por horário. Gerenciamento da demanda. Comportamento dos usuários. Transporte público.

\section{Abstract}

One of the biggest problems of medium and large size cities today is urban mobility. The solutions necessarily come through collective public transport systems, which require large investments in infrastructure. The collective public transport fleet is determined based on the number of passengers during the peak periods, and has an important role in determining the fare. The differentiation of fare based on the hour of the day, although still very new in Brazil, is a strategy widely used abroad to reduce

LR é engenheira civil pela Universidade Federal da Paraíba (UFPB) e mestra em engenharia civil com ênfase em Transportes e Gestão das Infraestruturas na Universidade Federal de Pernambuco (UFPE), e-mail: ligi.rabay@gmail.com

NPA é engenheiro civil e mestre em Geotecnia e Transportes pela Universidade Federal da Paraíba (UFPB) e doutor em Planejamento de Transportes pela University of Southampton, e-mail: niltonpereira.da@gmail.com 
demand during peak periods. The offer of discounts on fares during periods of lower demand can attract part of the users that usually travel during peak periods. As a result, this article presents a detailed study on the subject, with a viability analysis of the use of different fares based on the hour of the day, on the public transport system of a Brazilian city. The results show that 69\% of peak period users would be willing to change their traveling time to save money, and that the percentage of participation will vary depending on the size of the discount offered. Also, there is a minimum discount that would engage this change. The strategy seems viable, with good chances of changing the demand variation profile throughout the day and contributing to a reduction in the fleet and the system's operating costs.

Keywords: Peak-load pricing. Demand management. User behavior. Public transport.

\section{Introdução}

A mobilidade urbana tem sido indiscutivelmente um dos principais problemas das gestões municipais nas grandes e médias cidades brasileiras. As medidas recomendadas para lidar com esse tema envolvem investimentos em infraestrutura viária, priorização do transporte público, restrição ao uso do transporte individual e incentivo à utilização de meios de transporte não motorizados. São medidas que envolvem disputa por espaços urbanos limitados e recursos financeiros, na maioria das vezes, não disponíveis.

Há uma visão consensual hoje de que a solução para o problema passa necessariamente pelo sistema de transporte público coletivo, cujo acesso e eficiência dependem muito das políticas tarifárias adotadas. O Sistema de Informações da Mobilidade Urbana da ANTP (2014) mostra que, nos municípios brasileiros com população igual ou superior a 60 mil habitantes, $29 \%$ dos deslocamentos diários são feitos por transporte público, dos quais $25 \%$ são realizados por ônibus, e $4 \%$, por trilhos. Um estudo realizado por Dandapat et al. (2017) traz evidências que questionam a abordagem convencional do aumento da tarifa de ônibus para satisfazer a viabilidade operacional no contexto de um país emergente. Os autores expõem que a tarifa e a receita são fatores que estão sendo distorcidos devido ao excesso de oferta e a uma deficiência no serviço, por isso seria necessário melhorar o serviço de ônibus urbano por meio da regulamentação e do gerenciamento dos fatores ligados à operação do transporte para que não haja a necessidade de encargos financeiros adicionais aos usuários.

Uma das estratégias bastante utilizadas no exterior é a diferenciação do valor da tarifa por faixa horária, visando diminuir a oscilação da demanda ao longo do dia por meio da transferência de parte dos usuários que viajam nos períodos de pico para os períodos fora de pico, o que é feito por meio de descontos no valor da tarifa para os horários de menor demanda (Liu \& Charles, 2013; Rantzien \& Rude, 2014). Trata-se de uma alternativa que não necessita de grandes modificações no sistema já existente e pode gerar bons resultados tanto no uso mais racional da oferta quanto no melhor gerenciamento da demanda ao longo do dia.

0 artigo relata um estudo cujo objetivo foi avaliar a viabilidade do uso de tarifa diferenciada por horário no sistema de transporte público da cidade de João Pessoa/PB, investigando a predisposição dos usuários para aderir as mudanças no horário das viagens mediante desconto no valor da tarifa, a dimensão dessa possível adesão e os seus impactos na programação da oferta do serviço nos períodos de pico.

A estruturação do trabalho é organizada em nove seções: a primeira é esta etapa introdutória; a segunda e a terceira seções apresentam a relação de demanda e de tarifa e explanam os casos dos transportes públicos no exterior e no Brasil; a quarta seção expõe as características do local de estudo; a quinta, a sexta e a sétima seções apresentam a metodologia, a coleta e a análise de dados; e as duas últimas seções expõem os principais resultados e as discussões, bem como as considerações finais e as recomendações, respectivamente. 


\section{Relação entre demanda e tarifa}

Apesar de ser um serviço essencial para a população, vários fatores interferem nas decisões dos consumidores sobre o uso do transporte público. 0 valor da tarifa, a classe social do usuário, as características da viagem, a qualidade do serviço, o custo de serviços semelhantes substitutos e as condições meteorológicas são alguns exemplos de fatores que afetam essa demanda (Litman, 2007). A tarifa cobrada é indispensável para a oferta do serviço, uma vez que é por meio dela que são criadas as receitas das operadoras, e o seu impacto na quantidade de usuários é relativamente fácil de verificar (Albalate \& Bel i Queralt, 2009).

Sendo a demanda desse serviço sensível às variações do valor da tarifa, medir o comportamento dessa demanda e, consequentemente, da receita em relação à variação das tarifas é de especial interesse dos planejadores do serviço. Os reflexos na demanda decorrentes de flutuações nos preços são medidos na economia pela elasticidade-preço da demanda, quando se relaciona a variação percentual da quantidade com a variação percentual do preço (Carvalho, 2012). Em outras palavras, a elasticidade expressa a capacidade de resposta dos usuários às mudanças no preço.

De acordo com a teoria macroeconômica, a quantidade demandada de determinado produto depende, dentre outros fatores, do preço desse produto. Sendo mantidos estáveis todos os outros fatores, a elevação do preço de um produto ou serviço, como o aumento da tarifa, implica uma diminuição da quantidade demandada, isto é, da demanda.

Diversos estudos têm abordado a questão da elasticidade-preço da demanda do serviço de transporte público, ou seja, a sensibilidade da demanda em relação às oscilações no preço da tarifa.

Podem ser citados: McGeehan (1984), que estudou o comportamento da demanda de passageiros de trem intermunicipal na Irlanda no período de 1970 a 1982; Fowkes et al. (1985), que analisaram dados anuais de 1972 a 1981 das dez maiores rotas do Reino Unido; Owen \& Phillips (1987), que examinaram a demanda de passageiros por trem no Reino Unido em deslocamentos intermunicipais; Goodwin (1992), que analisou 50 estudos envolvendo elasticidade-preço da demanda em sistemas de ônibus; Bresson et al. (2004), que realizaram estudo em 62 áreas da França com dados do período de 1975 a 1995; Carvalho \& Pereira (2011), que investigaram o comportamento da demanda com relação ao valor da tarifa e nível de renda nos sistemas de transporte de nove capitais brasileiras; Liu \& Charles (2013), que relataram um estudo baseado em 77 casos de aumento de tarifas ao longo de duas décadas nos Estados Unidos; Holmgren (2013), que pesquisou a demanda do sistema de trens e ônibus na Suécia; e Oliveira et al. (2015), que realizaram um estudo sobre a demanda do transporte coletivo urbano na Região Metropolitana de São Paulo (RMSP).

Todos esses estudos demonstraram a sensibilidade da demanda em relação às variações da tarifa e que o aumento no valor da passagem paga pelo usuário provoca redução na quantidade de passageiros transportados.

De acordo com Bresson et al. (2004), a alteração no valor da tarifa é a forma mais direta e poderosa de influenciar a demanda de passageiros de um sistema de transporte público. No entanto, Litman (2004) enfatiza que a natureza das variações entre demanda e valor da tarifa depende de especificidades locais, como condições geográficas, cultura, tipo de usuário, demografia e intervalo de tempo considerado. Assim, é necessário que sejam desenvolvidos estudos para investigar a elasticidade específica de cada localidade.

Uma vez que as tarifas de transporte público são definidas a partir dos custos operacionais e que os custos nos horários de pico são muito mais elevados do que fora de pico, estratégias que diminuam as diferenças entre demandas nesses períodos, suavizando-as no decorrer do dia, podem representar uma importante contribuição para a redução dos custos operacionais e, consequentemente, do valor da tarifa (Rantzien \& Rude, 2014).

O mesmo posicionamento é compartilhado por Hounsell (1991). Para ele, a diferenciação de preços pode ser utilizada como instrumento de suavização da demanda ao longo do dia, seja aumentando a tarifa no horário de pico, seja oferecendo descontos no período fora de pico, seja uma combinação das medidas. 


\section{Tarifa diferenciada nos transportes públicos}

No setor dos transportes públicos urbanos, a diferenciação tarifária é utilizada em muitas cidades no exterior, mas ainda incipiente no Brasil, e é um tema muito abordado como solução para várias cidades devido aos resultados satisfatórios obtidos onde foi adotada. Porém, mesmo com esses resultados, sua implantação costuma gerar questionamentos e entraves jurídicos com as Procuradorias do Consumidor e o Ministério Público pela cobrança de valores diferenciados pelo mesmo serviço (NTU, 2005).

Apesar dos questionamentos, existem estudos que expõem a necessidade de buscar soluções para as cidades brasileiras. Um deles é o de Antunes \& Simões (2013), realizado em três cidades brasileiras de porte médio, que indica que o atributo que mais produz insatisfação geral é a lotação, com maior evidência nos horários de pico, revelando a necessidade de alguma estratégia de gerenciamento dessa demanda. A essa mesma conclusão chegaram Suman et al. (2017), no estudo sobre serviço de ônibus de Deli e Mumbai, na Índia, o qual revelou que a superlotação é a grande preocupação dos usuários dos sistemas das duas cidades, e Li \& Hensher (2013), na pesquisa envolvendo o trem de passageiros de Sydney e Melbourne, na Austrália, segundo a qual o nível de lotação é equivalente ao tempo de viagem na decisão do usuário sobre o uso do transporte público. Outro estudo realizado no metrô de Paris, na França, no horário de pico mostrou que a lotação afeta a satisfação dos usuários pelo desconforto físico, pelo impedimento de se sentar durante a viagem, pela perda de tempo (não podem utilizar o tempo do trajeto de forma útil), pelo incômodo e pela proximidade física com as outras pessoas (Haywood et al., 2017).

\section{Experiências no exterior}

Na América Latina, uma referência no transporte público em se tratando da aplicação dessa estratégia é Santiago, no Chile. A tarifa diferenciada por horário foi implantada no metrô e nos ônibus da cidade com três preços distintos, como mostra a Tabela 1. São utilizadas três faixas horárias: pico, fora de pico ou baixa.

\begin{tabular}{|c|c|c|c|}
\hline Período & Serviço & Horário & Tarifa (\$) \\
\hline Pico & $\begin{array}{c}\text { Metrô } \\
\text { Ônibus e metrô }\end{array}$ & $\begin{array}{l}\text { 07:00-09:00 } \\
\text { 18:00-20:00 }\end{array}$ & 740 \\
\hline Fora de pico & $\begin{array}{c}\text { Metrô } \\
\text { Ônibus e metrô }\end{array}$ & $\begin{array}{l}06: 30-07: 00 \\
09: 00-18: 00 \\
20: 00-20: 45\end{array}$ & 660 \\
\hline Baixa & $\begin{array}{c}\text { Metrô } \\
\text { Ônibus e metrô }\end{array}$ & $\begin{array}{l}06: 00-06: 30 \\
20: 45-23: 00\end{array}$ & $\begin{array}{l}610 \\
640\end{array}$ \\
\hline
\end{tabular}

Fonte: Metro de Santiago (2016).

No serviço de metrô, a tarifa no horário de pico é \$ 740 pesos, enquanto fora de pico, \$ 660 pesos, o que representa um desconto de $10,81 \%$. Já no período de baixa, a tarifa cobrada é $\$ 610$ pesos, ou seja, $17,57 \%$ menor. Assim, o usuário que utiliza o metrô no período de pico pode ter uma redução de $\$ 80$ pesos fora de pico ou de $\$ 130$ pesos no período de baixa (Metro de Santiago, 2016).

Para o usuário que usa os serviços combinados de ônibus e metrô no horário de pico, a tarifa é de $\$ 740$ pesos, enquanto nos períodos fora de pico e de baixa, de $\$ 660$ pesos e $\$ 640$ pesos, respectivamente. Ou seja, a economia é de $\$ 80$ pesos para o período fora de pico e $\$ 100$ para o período de baixa. Nos sábados, domingos e feriados, a tarifa cobrada é a correspondente à tarifa fora de pico (Metro de Santiago, 2016).

Outra cidade que utiliza de forma integrada a tarifação diferenciada é Londres, na Inglaterra. 0 sistema de metrô possui uma tarifa diferenciada de acordo com as zonas da cidade, a forma de pagamento (cartão ou dinheiro) e o horário de utilização (pico ou fora de pico). Os horários de pico 
considerados são apenas nos dias úteis. A Tabela 2 mostra a variação existente entre as tarifas de acordo com as zonas e o período do dia, para pagamento com cartão no metrô. 0 pico da manhã é das $7 \mathrm{~h}$ às $10 \mathrm{~h}$ e o da tarde é das $16 \mathrm{~h}$ às $19 \mathrm{~h}$. Os demais horários são considerados fora de pico.

Tabela 2 - Tarifas do sistema de metrô de Londres

\begin{tabular}{cccc}
\hline \multirow{2}{*}{ Zonas da cidade } & \multicolumn{2}{c}{ Tarifa $(\boldsymbol{(})$} & \multirow{2}{*}{ Desconto (\%) } \\
\cline { 2 - 3 } & Pico & Fora de pico & \\
\hline $1-1$ & 2.40 & 2.40 & - \\
$1-2$ & 2.90 & 2.40 & 17,24 \\
$1-3$ & 3.30 & 2.80 & 15,15 \\
$1-4$ & 3.90 & 2.80 & 28,21 \\
$1-5$ & 4.70 & 3.10 & 34,04 \\
$1-6$ & 5.10 & 3.10 & 39,22 \\
\hline
\end{tabular}

Fonte: Transport for London (2016).

Para pagamento com cartão, os descontos da zona 1 para as demais zonas variam de 15,15 a 39,22\%. Para pagamento em dinheiro, as tarifas variam de $€ 4,90$ a $€ 6,00$, e não há desconto.

Estudos têm sido realizados para analisar a possibilidade de implantação dessa estratégia, a aceitação por parte dos usuários, das empresas e dos órgãos públicos e as vantagens socioeconômicas referentes a essa modificação. Bianchi et al. (1998) desenvolveram um estudo no metrô de Santiago para avaliar o impacto dos diferentes preços em cada período na mudança de horário das viagens. Os resultados mostram que os usuários mudariam seus horários de viagem mediante a existência de um desconto na tarifa, além de um aumento do conforto, verificando que existe um comportamento bem próximo do que há no sistema.

Outro trabalho, conduzido por Rantzien \& Rude (2014), analisou o caso do sistema de Estocolmo, na Suécia, com objetivo de investigar como o valor da tarifa afeta a demanda por transporte público nos horários de pico e fora de pico. A análise levou à conclusão de que o aumento do valor da tarifa no período de pico e a sua redução no período fora de pico geram aumento da receita e do número de passageiros no âmbito global, bem como diminuição da demanda no horário de pico.

\section{Experiências no Brasil}

Ferronatto (2002) desenvolveu um estudo como objetivo de obter informações sobre a sensibilidade dos usuários de transporte coletivo à diferenciação tarifária por hora do dia, além de avaliar o seu impacto nos custos de operação. Os resultados mostram que essa política tinha potencial para influenciar a demanda, diluindo os picos. Com objetivo semelhante, Feres (2015) estimou a demanda do transporte público a partir de uma redução $10 \%$ no valor da tarifa nos horários fora de pico e concluiu que essa medida resultou em uma diminuição de 6,5\% no número de passageiros no horário de pico e uma elevação de $20,5 \%$ no vale (fora do pico). Por outro lado, o estudo mostrou também que o aumento da tarifa do horário de pico leva a uma suavização mais aguda, mas destaca a possibilidade de haver evasão de passageiros em vez de suavização de demanda.

Em Londrina/PR, foi aprovada a diferenciação de tarifa no transporte público da cidade em janeiro de 2016, e as pessoas que utilizassem o transporte em horários fora de pico teriam um desconto de até 10\% na tarifa (Frazão, 2015).

Outro exemplo de aplicação é a cidade de Fortaleza/CE, onde a variação é adotada no sistema de ônibus e vans desde 2011 e foi intitulada "Hora Social" (Tabela 3). 0 valor da tarifa para os serviços fora do pico é 7,27\% menor para quem paga inteira e 7,69\% para estudantes. Além disso, o sistema também possui a "Tarifa Social", que ocorre aos domingos e em dias especiais (ETUFOR, 2016). 
Tabela 3 - Tarifas do sistema de transporte público de Fortaleza

\begin{tabular}{ccccc}
\hline \multirow{2}{*}{ Tipo de tarifa } & Dias da semana & \multirow{2}{*}{ Horário } & \multicolumn{2}{c}{ Valor (R\$) } \\
\cline { 3 - 5 } & Segunda a sábado & Demais horários & 2,75 & 1,3 \\
\hline Convencional & Segunda a sábado & 9h às 10h & Meia \\
Hora social & 15h às 16h & 2,55 & 1,2 \\
Tarifa social & Domingos, 13 de abril, Réveillon e 1 ${ }^{\circ}$ de janeiro & Todos os horários & 2,15 & 1 \\
\hline
\end{tabular}

Fonte: Empresa de Transporte Urbano de Fortaleza (ETUFOR, 2016).

Em São Paulo/SP, a Assembleia Legislativa aprovou no início de 2016 o Projeto de Lei no 1.442, ainda não sancionado pelo governador, para a redução da tarifa de transporte público fora do horário de pico. A proposta busca incentivar o uso de transportes públicos (ônibus, metrô, trens e trólebus) e, consequentemente, contribuir para a redução dos congestionamentos provocados pelo grande uso do transporte privado (São Paulo, 2015).

\section{Estudo de caso}

A cidade objeto do estudo foi João Pessoa/PB, que possui em torno de 800 mil habitantes. Seu sistema de transporte público coletivo possui uma rede estruturada a partir de sete corredores principais que convergem para área central, com o Terminal de Integração do Varadouro (central), além de seis outros terminais de integração distribuídos na periferia. Possui também bilhetagem eletrônica e integração temporal, inclusive com a rede metropolitana. A Tabela 4 contém outras informações sobre o sistema de transporte coletivo da cidade (SEMOB/JP, 2016).

\begin{tabular}{|c|c|}
\hline Linha de ônibus & 101 linhas \\
\hline Frota operacional & 470 ônibus \\
\hline Idade média da frota & 4,3 anos \\
\hline Passageiros transportados/mês & 8.438 .995 \\
\hline Média de viagem dos ônibus/dia & 4.875 \\
\hline Índice de passageiros/quilômetro (IPK) & 1,72 (média em 12 meses) \\
\hline Tarifa & $R \$ 3,00$ \\
\hline
\end{tabular}

Fonte: SEMOB/JP (2016).

\section{Metodologia}

O estudo foi desenvolvido conforme as etapas descritas. Primeiro, foi necessária a determinação dos períodos de pico do sistema de transporte público da cidade por meio do número diário de passageiros transportados em intervalos de 15 minutos. Esses dados foram disponibilizados pela Superintendência Executiva de Mobilidade Urbana de João Pessoa (SEMOB/JP) para os dias 8 (terça-feira), 9 (quarta-feira) e 10 (quinta-feira) de março de 2016.

A pesquisa de campo teve como objetivo principal determinar, entre os passageiros que utilizam o transporte público no período de pico, aqueles que estariam dispostos a mudar de horário caso existisse uma diferenciação tarifária e qual seria o valor aceitável para essa mudança. A pesquisa foi feita por meio de uma amostragem por conveniência.

0 dimensionamento da amostra ocorreu a partir de uma margem de erro e um grau de confiança predeterminados, e a coleta se deu em pontos estratégicos de passagem de pessoas, como terminais e estações de transferência, em horários predefinidos.

Uma pesquisa teste foi realizada para avaliar o processo de coleta, os locais selecionados e o questionário elaborado. A análise da amostra obtida e a expansão dos resultados permitiram estimar as características 
predominantes da população usuária e a redução do número de passageiros, viagens e frota de veículos referentes a um grupo de linhas, segundo procedimentos encontrados em Ferraz \& Torres (2004).

Por último, foram analisados os resultados obtidos em termos de impactos na demanda e oferta do serviço de transporte público estudado, assim como a viabilidade do uso da estratégia.

\section{Coleta de dados}

No estudo, foram utilizados dados operacionais obtidos diretamente do órgão gestor e da pesquisa de campo realizada com os usuários do sistema.

\section{Horários de pico do sistema}

Com os dados fornecidos pela SEMOB/JP para os três dias úteis, foram determinadas as horas de pico e de vale (fora de pico) para cada turno. Uma vez que o objetivo do uso de diferentes tarifas é diminuir a demanda no pico e aumentar a do vale, suavizando-as no decorrer do dia, a média dessas demandas foi utilizada como referência para identificar os intervalos de pico a serem considerados no estudo, os quais se encontram na Tabela 5 e são ilustrados na Figura 1.

Tabela 5 - Determinação dos picos para a média dos três dias

\begin{tabular}{ccccc}
\hline Pico & Máx. (pass.) & Mín. (pass.) & Média (pass.) & Horário \\
\hline Manhã & 26.177 & 15.261 & 20.719 & $05: 45$ às 07:45 \\
Tarde & 25.898 & 17.138 & 21.518 & $15: 45$ às 18:00 \\
\hline
\end{tabular}

Fonte: SEMOB/JP (2016).

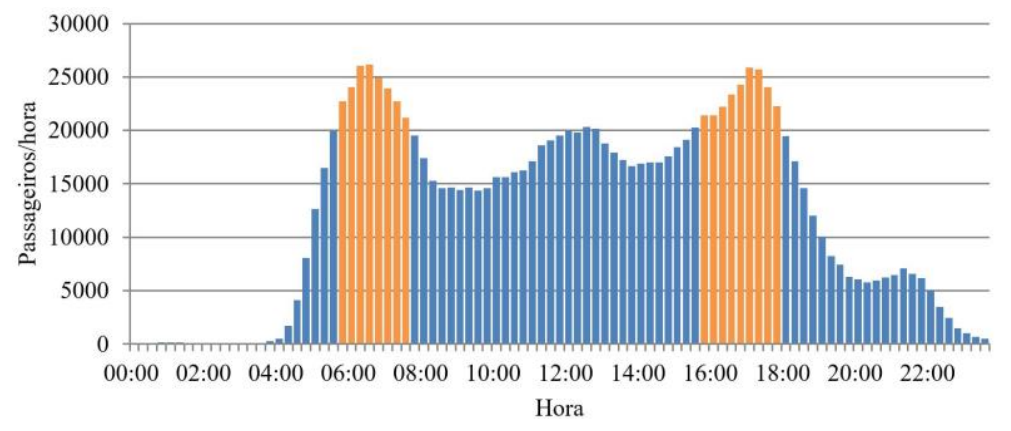

Figura 1 - Variação da demanda diária média para os três días. Fonte: SEMOB/JP (2016).

Constatou-se que os horários de pico ocorriam das $5 \mathrm{~h} 45$ às $7 \mathrm{~h} 45$ e das $15 \mathrm{~h} 45$ às $18 \mathrm{~h} 00$. Esses horários foram arredondados para os horários inteiros mais próximos (das $6 \mathrm{~h}$ às $8 \mathrm{~h}$ e das $16 \mathrm{~h}$ às $18 \mathrm{~h}$ ) para facilitar a execução da pesquisa e a compreensão dos usuários.

\section{Definição da amostra}

A determinação da amostra foi feita por meio dos estudos não probabilísticos. Como foi realizada mediante abordagem de pessoas em pontos de grande movimentação de usuários do transporte público, a amostragem por conveniência se tornou mais viável. Assim, partindo-se da adoção de um grau de confiança de 95\%, um erro absoluto de 5\% e uma população de aproximadamente 90 mil passageiros por dia no pico, a amostra necessária seria de 383 usuários, sendo adotado um valor em torno de 400 entrevistas para o estudo. 


\section{Estruturação do formulário}

A pesquisa foi estruturada de modo a obter todas as informações necessárias de forma rápida e de fácil entendimento para a população abordada. As informações fundamentais para o estudo foram: horário e frequência de utilização do transporte público; motivo da viagem; possibilidade de modificação de horário; e desconto mínimo necessário que motivasse a mudança.

\section{Realização do teste}

Uma pesquisa teste foi realizada com a finalidade de definir melhor a forma de contato com os usuários, identificar as dificuldades e testar os pesquisadores e sua forma de abordagem.

0 primeiro ponto observado nessa fase foi a forma de contato com os entrevistados. Uma alternativa que facilitou a compreensão rápida das questões foi a apresentação verbal de exemplos. Também foram definidos, a partir da realização do teste, os dias e os locais da pesquisa. Foram excluídas a manhã de segunda-feira e a tarde de sexta-feira. Para alcançar um maior número de pessoas em cada um dos horários, os locais de pesquisa selecionados foram o Terminal de Integração do Varadouro no pico da manhã e as estações de embarque/desembarque do parque Sólon de Lucena no pico da tarde, no qual existe grande fluxo de passageiros por ser também na área central da cidade.

\section{Análise dos dados}

A realização da pesquisa ocorreu no final de abril e início de maio de 2016, quando foram obtidas 395 entrevistas válidas.

\section{Caracterização geral}

O público-alvo demonstrou ser bastante dependente do sistema: $52 \%$ dos entrevistados não possuíam qualquer meio de locomoção privado, sendo indispensável o uso do transporte público. Por outro lado, o restante informou possuir carro, moto ou os dois em sua residência, porém não possuía condições financeiras de utilizá-los nos deslocamentos diários. A grande maioria (86\%) realizava três ou mais viagens no sistema por semana.

Os motivos dessas viagens estão ilustrados na Figura 2, no qual se observa que $89 \%$ das razões dos deslocamentos são os chamados "obrigatórios" (trabalho e estudo), que possuem horários fixos de início e término.

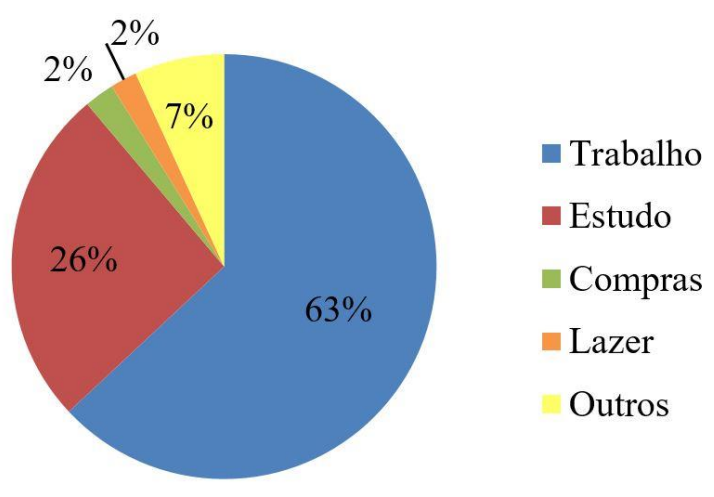

Figura 2 - Motivos da viagem. Fonte: elaborado pelo autor (2016). 


\section{Possibilidade de mudança de horário}

Quando questionados sobre a possibilidade de mudança no horário do deslocamento mediante a oferta de um desconto no valor da tarifa, as respostas foram as que se encontram na Figura 3. Assim, foi constatado que $69 \%$ dos usuários estariam dispostos a mudar seus horários de viagens em troca de uma redução no valor da tarifa. Além disso, desses 69\%, 39\%, mesmo tendo dependência da utilização do transporte público nos horários de pico devido ao deslocamento obrigatório, possuíam a disposição de reorganizar o horário de deslocamento para obter algum benefício financeiro.

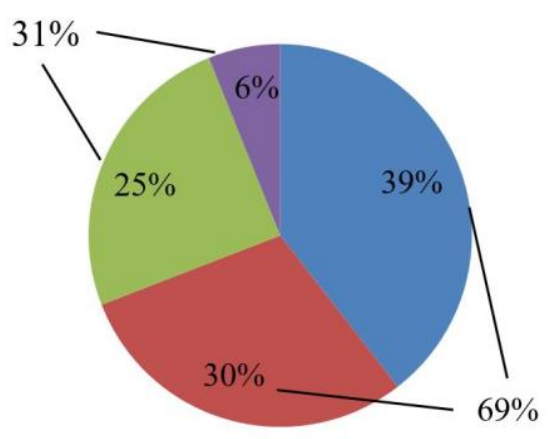

$$
\begin{aligned}
& \text { norário, mas precisam } \\
& \text { ' } \\
& \text { SIM - Mudariam de } \\
& \text { horário e não precisam } \\
& \text { NÃO - Não mudariam de } \\
& \text { horário porque precisam } \\
& \text { NÃO - Não mudariam de } \\
& \text { horário, mas não precisam }
\end{aligned}
$$

Figura 3 - Possibilidade de mudança de horario. Fonte: elaborado pelo autor (2016).

\section{Identificação do desconto aceitável}

A finalidade desse questionamento foi identificar o desconto a partir do qual o usuário estaria disposto a mudar o horário das viagens realizadas atualmente e passar para fora do período de pico. Foi necessário esclarecer que o desconto deveria ser o menor valor que motivasse a mudança, sem induzir a escolha dos entrevistados. Também foi importante fazer referência ao valor reduzido da tarifa, uma vez que a maioria não assimilava bem o desconto em termos percentuais ou o próprio valor do desconto. A Figura 4 mostra os resultados obtidos.

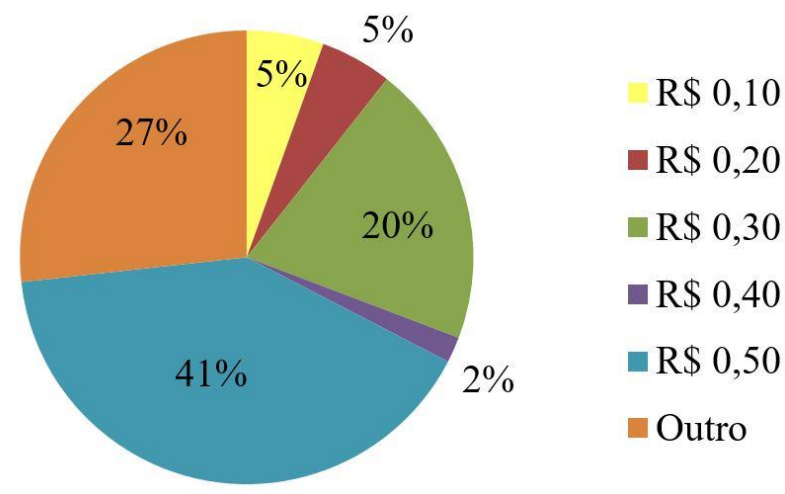

Figura 4 - Desconto aceitável. Fonte: elaborado pelo autor (2016).

Como era de se esperar, a disponibilidade para mudança de horário aumentou à medida que o valor da tarifa era reduzido. Descontos de $\mathrm{R} \$ 0,10$ e 0,20 não se mostraram muito atraentes, com apenas $5 \%$ de adesão dos usuários em cada caso. Porém, para um desconto de $\mathrm{R} \$ 0,30$, correspondente a $10 \%$ do valor da tarifa vigente, o percentual de adesão foi de $20 \%$. Já para uma redução de $\mathrm{R} \$ 0,40$, o percentual de aceitação da mudança foi surpreendentemente baixo, apenas $2 \%$. 0 percentual de $41 \%$ foi, naturalmente, para o maior valor apresentado, $\mathrm{R} \$ 0,50$. 
O usuário que se dispõe a mudar de horário por um desconto de $\mathrm{R} \$ 0,10$ ou 0,20 obviamente o faria por $\mathrm{R} \$ 0,30$. Assim sendo, para um desconto de $\mathrm{R} \$ 0,30$, o percentual total de usuários que estaria disposto a mudar de horário seria $30 \%$, que é bastante expressivo. 0 aumento de mais $\mathrm{R} \$ 0,10$ muito pouco acrescentaria em termos de transferência de demanda.

É importante ressaltar que esses resultados tiveram como base apenas os usuários que afirmaram estar dispostos a modificar seus horários mediante um desconto no valor da tarifa, ou seja, os $69 \%$ identificados na Figura 3.

\section{Redução da demanda no pico}

A partir dos dados de demanda disponibilizados pela SEMOB/JP, a quantidade de passageiros que seria reduzida em cada período de pico para cada desconto foi determinada com base nos percentuais contidos na Figura 4, fazendo o ajuste necessário para a amostra em questão, a qual foi de $69 \%$ do total dos entrevistados. 0 resultado final foi obtido a partir desse percentual sobre a quantidade de usuários do sistema em cada período de pico.

Segundo a SEMOB/JP, eram transportados, em média, 44.444 passageiros no pico da manhã e 46.526 passageiros no pico da tarde. Assim, aplicando-se os percentuais encontrados sobre as demandas de cada período de pico, tem-se a estimativa do número de passageiros que migraria para os períodos fora de pico, como mostra a Tabela 6.

Tabela 6 - Estimativa da redução da demanda no pico

\begin{tabular}{ccccc}
\hline \multirow{2}{*}{ Desconto mínimo } & \multirow{2}{*}{ Desconto (\%) } & \multirow{2}{*}{ Correção (\%) } & \multicolumn{2}{c}{ Redução de passageiros } \\
\cline { 4 - 5 } & & & Manhã & Tarde \\
\hline $\mathrm{R} \$ 0,10$ & 5 & 3,45 & 1.533 & 1.605 \\
$\mathrm{R} \$ 0,20$ & 10 & 6,90 & 3.067 & 3.210 \\
$\mathrm{R} \$ 0,30$ & 30 & 20,70 & 9.200 & 9.631 \\
$\mathrm{R} \$ 0,40$ & 32 & 22,08 & 9.813 & 10.273 \\
$\mathrm{R} \$ 0,50$ & 73 & 50,37 & 22.386 & 23.435 \\
\hline
\end{tabular}

Fonte: elaborada pelo autor (2016).

\section{Redução do número de viagens}

O número de viagens em determinada linha aumenta à medida que a demanda sobe. A redução das viagens, no entanto, só pode ocorrer até certo patamar, pois uma oferta mínima deve ser assegurada à população. Nos períodos de pico, essa relação é de tal forma que a diminuição na demanda tem reflexos equivalentes no número de viagens. Partindo desse princípio e com base nos dados fornecidos pela SEMOB/JP, que foram de 492 viagens no pico da manhã e 483 viagens no pico da tarde, estimou-se o número de viagens que seriam eliminadas em decorrência da redução da demanda, como mostra a Tabela 7.

\begin{tabular}{ccc} 
Tabela 7 - Redução do número de viagens \\
\hline Desconto & \multicolumn{2}{c}{ Número de viagens reduzidas } \\
\cline { 2 - 3 } Mínimo & Manhã & Tarde \\
\hline$R \$ 0,10$ & 17 & 17 \\
$R \$ 0,20$ & 34 & 33 \\
$R \$ 0,30$ & 102 & 100 \\
$R \$ 0,40$ & 109 & 107 \\
$R \$ 0,50$ & 248 & 243 \\
\hline
\end{tabular}

Fonte: elaborada pelo autor (2016). 
Porém, esse procedimento não é muito preciso, uma vez que não leva em conta as particularidades de cada linha. Assim sendo, foram coletados dados específicos de algumas linhas para fins de dimensionamento e, em seguida, determinada a redução de frota para cada caso.

A estimativa da redução percentual dos custos pode ser feita para cada linha a partir da diminuição proporcional das viagens e da frota operante, decorrente da alteração do número de usuários da linha estudada.

Inicialmente, foi realizada uma pesquisa de carregamento durante o período de pico para obtenção de uma amostra de cada linha. Com a expansão da amostra, foi possível estimar o total de passageiros no período de pico, a demanda da seção crítica, o número de viagens e a frota necessária. Considerando as reduções nas demandas de cada linha devido à transferência dos usuários proporcionada pelos descontos oferecidos, foram dimensionadas as viagens e a frota para esse novo cenário.

Essa análise específica foi realizada em seis linhas de diferentes corredores da cidade: 204-Cristo Redentor, 302-Cidade Verde/Pedro II, 401-Altiplano, 506-Bairro dos Estados, 511-Tambaú/Manaíra Shopping e 521-Tambaú/Hiper Bompreço. Está detalhada neste artigo a linha 204-Cristo Redentor a título de exemplo.

\section{Linha 204-cristo redentor}

A linha 204 é responsável pelo deslocamento de usuários do bairro do Cristo Redentor até o Terminal de Integração do Varadouro no centro da cidade. A Tabela 8 resume os resultados do redimensionamento dessa linha, considerando veículos com capacidade para 77 passageiros e um tempo de ciclo de 56 minutos.

Tabela 8 - Redimensionamento da linha 204

\begin{tabular}{cccccc}
\hline Desconto (RS) & $\begin{array}{c}\text { Redução da } \\
\text { demanda (\%) }\end{array}$ & $\mathbf{P}$ & $\mathbf{Q}$ & $\mathbf{H}$ & $\mathbf{F}$ \\
\hline- & 0,00 & 676 & 9 & 13 & 4 \\
0,10 & 3,45 & 653 & 8 & 15 & 4 \\
0,20 & 6,90 & 630 & 8 & 15 & 4 \\
0,30 & 20,70 & 537 & 7 & 17 & 3 \\
0,40 & 22,08 & 527 & 7 & 17 & 3 \\
0,50 & 50,37 & 336 & 4 & 30 & 2 \\
\hline
\end{tabular}

Em que: $P=$ demanda na seção crítica (pass. $/ h$ ); $Q$ = número de viagens na linha para atender à demanda (viag./h); $H=$ intervalo entre viagens (min./veíc.); $\mathrm{F}=$ frota necessária (veíc.). Fonte: elaborada pelo autor (2016).

A partir desses números, observa-se a relação direta que existe entre o valor do desconto oferecido e seus reflexos no número de viagens e frota necessária. A Tabela 9 mostra os resultados obtidos, por meio do mesmo procedimento, para as outras linhas estudadas.

Tabela 9 - Resultados da redução da frota

\begin{tabular}{cccccc}
\hline \multirow{2}{*}{ Redução (R\$) } & \multicolumn{5}{c}{ Frota das linhas } \\
\cline { 2 - 6 } & $\mathbf{3 0 2}$ & $\mathbf{4 0 1}$ & $\mathbf{5 0 6}$ & $\mathbf{5 1 1}$ & $\mathbf{5 2 1}$ \\
\hline- & 6 & 5 & 2 & 6 & 4 \\
0,10 & 6 & 4 & 2 & 6 & 4 \\
0,20 & 6 & 4 & 2 & 6 & 4 \\
0,30 & 5 & 4 & 2 & 5 & 3 \\
0,40 & 5 & 4 & 2 & 4 & 3 \\
0,50 & 3 & 2 & 1 & 3 & 2 \\
\hline
\end{tabular}

Fonte: elaborada pelo autor (2016). 


\section{Resultados e discussões}

O conhecimento da demanda do sistema de transporte público urbano de João Pessoa e a realização da pesquisa de campo permitiram obter as respostas desejadas no início do estudo. De acordo com a amostra coletada, $86 \%$ dos usuários utilizavam três ou mais vezes por semana e $52 \%$ dependiam exclusivamente do sistema para seus deslocamentos. Com relação ao uso da estratégia da diferenciação tarifária e à aceitação dos entrevistados, 69\% afirmaram estar dispostos a mudar o horário da viagem para obter alguma economia. Os descontos apresentados variaram de $R \$ 0,10$ a $R \$ 0,50$, equivalente a 3,33 e $16,67 \%$ do valor vigente da tarifa, respectivamente, que levaram de 3,5 a 50,4\% dos usuários a manifestar interesse em modificar seus horários de viagem.

A aplicação dos percentuais de redução da demanda diretamente nas viagens do sistema permitiu determinar uma redução entre 17 e 248 viagens. Analisando as linhas individualmente, observa-se que, em quatro linhas pesquisadas, a redução da frota ocorreu quando o desconto oferecido foi a partir de $\mathrm{R} \$ 0,30$ e que para uma delas, a 506, a mudança não ocorreu devido ao fato de possuir apenas dois veículos no período de pico, o que, por si só, já é muito baixo. A Tabela 10 mostra os percentuais de redução da frota relacionados a cada linha de ônibus para o desconto de $\mathrm{R} \$ 0,30$.

\begin{tabular}{|c|c|c|c|}
\hline Himh & \multicolumn{2}{|c|}{ Frota (veíc.) } & \multirow{2}{*}{ Redução (\%) } \\
\hline LInna & Calculada & Reduzida & \\
\hline 204 & 4 & 3 & 25,0 \\
\hline 302 & 6 & 5 & 20,0 \\
\hline 401 & 5 & 4 & 20,0 \\
\hline 506 & 2 & 2 & 0,00 \\
\hline 511 & 6 & 5 & 20,0 \\
\hline 521 & 4 & 3 & 25,0 \\
\hline TOTAL & 27 & 22 & 18,52 \\
\hline
\end{tabular}

Fonte: elaborada pelo autor (2016).

0 percentual médio de redução da frota analisando as linhas individualmente $(18,52 \%)$ ficou relativamente próximo ao percentual de redução da demanda $(20,7 \%)$. Essa diferença fica ainda menor se, das linhas consideradas, forem excluídas aquelas que possuem uma frota muito baixa, como é o caso da linha 506. Nesse caso, a redução média das linhas ficaria em $20 \%$, o que é praticamente o percentual de redução da demanda (20,7\%). Com isso, é possível admitir que um desconto de $10 \%$ no valor da tarifa vigente poderia gerar uma redução de 20,7\% dos passageiros no horário de pico e, consequentemente, uma diminuição de aproximadamente $20 \%$ dos custos operacionais associados à frota do sistema nesse período.

\section{Considerações finais e recomendações}

O estudo desenvolvido permitiu constatar que a otimização de um sistema de transporte público pode ser feita sem grandes investimentos ou modificações em sua operação. A estratégia de diferenciação de tarifa ao longo do dia, bastante utilizada no exterior, ainda tem muito que ser explorada no Brasil. No caso específico do sistema de transporte público estudado, o de João Pessoa/PB, uma parcela considerável dos usuários se mostrou disposta a aceitar uma mudança nos horários de realização das suas viagens em troca de um desconto na tarifa. Uma redução de apenas $10 \%$ no valor da tarifa para quem viajar nos horários fora do pico seria suficiente para uma diminuição de aproximadamente $20 \%$ da demanda no horário de pico e uma redução nas mesmas proporções na frota operante. Assim, com uma frota operante menor, seria possível atender ao mesmo número diário de 
passageiros transportados, gerando uma redução nos custos fixos e variáveis e, consequentemente, a possibilidade de adoção de tarifas menores.

A pesquisa desenvolvida mostrou que a diferenciação tarifária por faixa horária se apresenta como uma opção viável e com boas chances de modificar o perfil da variação da demanda ao longo do dia. Porém, mais estudos se fazem necessários para um melhor conhecimento dos impactos dessa transferência de demanda para os períodos de vale, principalmente nos horários mais próximos aos períodos de pico, antes e depois, e sua viabilidade em termos econômicos, operacionais e, principalmente, de comodidade para o usuário.

\section{Referências}

Albalate, D., \& Bel i Queralt, G. (2009). Factors explaining urban transport systems in large European cities: a crosssectional approach (IREA-Working Papers, IR09/05). Barcelona: Universitat de Barcelona.

Antunes, E. M., \& Simões, F. A. (2013). Engenharia urbana aplicada: um estudo sobre a qualidade do transporte público em cidades médias. Urbe. Revista Brasileira de Gestão Urbana, 5(2), 51-62.

http://dx.doi.org/10.7213/urbe.05.002.SE04.

Associação Nacional das Empresas de Transportes Urbanos - NTU. (2005). Novas tendências em política tarifária. Brasília: NTU.

Associação Nacional dos Transportes Públicos - ANTP. (2014). Sistema de Informações da Mobilidade Urbana. (Relatório Geral, pp. 3-6). São Paulo. Recuperado em 14 de abril de 2018, de www.antp.org.br

Bianchi, R., Jara-Díaz, S. R., \& D. Ortúzar, J. (1998). Modelling new pricing strategies for the Santiago Metro. Transport Policy, 5(4), 223-232. http://dx.doi.org/10.1016/S0967-070X(98)00025-0.

Bresson, G., Dargay, J., Madre, J. L., \& Pirotte, A. (2004). Economic and structural determinants of the demand for public transport: an analysis on a panel of French urban areas using shrinkage estimators. Transportation Research Part A, Policy and Practice, 38(4), 269-285. http://dx.doi.org/10.1016/j.tra.2003.11.002.

Carvalho, C. H. R., \& Pereira, R. H. M. (2011). Efeitos da variação da tarifa e da renda da população sobre a demanda de transporte público coletivo urbano no Brasil (No. 1595, Texto para Discussão). Brasília: IPEA.

Carvalho, S. L. (2012). Análise de políticas tarifárias do transporte público do município de Belo Horizonte sob a ótica da elasticidade demanda-tarifa (Dissertação de mestrado). Universidade Federal de Minas Gerais, Belo Horizonte.

Dandapat, S., Cheranchery, M. F., \& Maitra, B. (2017). Is fare increment desirable for ensuring operational viability of private buses? Transport Policy, 59, 134-141. http://dx.doi.org/10.1016/j.tranpol.2017.07.010.

Empresa de Transporte Urbano de Fortaleza - ETUFOR. (2016). Transporte Regular (Ônibus) e Complementar (Vans). Recuperado em 13 de maio de 2016, de http://www.fortaleza.ce.gov.br/etufor/transporte-regular-onibuse-complementar-vans

Feres, L. J. (2015). Diferencial de tarifa entre pico e vale como ferramenta de suavização da demanda no sistema de transporte público da cidade de São Paulo (Dissertação de mestrado). Instituto de Ensino e Pesquisa, São Paulo.

Ferraz, A. C. P., \& Torres, I. G. E. (2004). Transporte público urbano (2a ed.). São Carlos: RiMa Editora.

Ferronatto, L. G. (2002). Potencial de medidas de gerenciamento da demanda no transporte público urbano por ônibus (Dissertação de mestrado). Universidade Federal do Rio Grande do Sul, Porto Alegre.

Fowkes, A. S., Nash, C. A., \& Whiteing, A. E. (1985). Understanding trends in Inter-city Rail Traffic in Great Britain. Transportation Planning and Technology, 10(1), 65-80. http://dx.doi.org/10.1080/03081068508717301.

Frazão, M. (2015). Londrina terá desconto na tarifa de ônibus fora do horário de pico. Jornal de Londrina. Recuperado em 28 de abril de 2016, de http://www.gazetadopovo.com.br/vida-e-cidadania/londrina-teradesconto-na-tarifa-de-onibus-fora-do-horario-de-pico-dxy7nlj0ko0pqbifphndublf2 
Goodwin, P. B. (1992). A review of new demand elasticities with special reference to short and long run effects of price changes. Journal of Transport Economics and Policy, 26(2), 155-169.

Haywood, L., Koning, M., \& Monchambert, G. (2017). Crowding in public transport: who cares and why? Transportation Research Part A, Policy and Practice, 100, 215-227. http://dx.doi.org/10.1016/j.tra.2017.04.022.

Holmgren, J. (2013). An analysis of the determinants of local public transport demand focusing the effects of income changes. European Transport Research Review, 5(2), 101-107. http://dx.doi.org/10.1007/s12544-013-00940 .

Hounsell, N. B. (1991). Peak spreading and congestion: techniques for distinguishing "passive" from "active" responses by road users. Transport Planning Systems, 1(3), 39-46.

Li, Z., \& Hensher, D. A. (2013). Crowding in public transport: a review of objective and subjective measures. Journal of Public Transportation, 16(2), 6. http://dx.doi.org/10.5038/2375-0901.16.2.6.

Litman, T. (2004). Transit price elasticities and cross-elasticities. Journal of Public Transportation, 7(2), 37-58. http://dx.doi.org/10.5038/2375-0901.7.2.3.

Litman, T. (2007). Developing indicators for comprehensive and sustainable transport planning. Transportation Research Record: Journal of the Transportation Research Board, 2017(1), 10-15. http://dx.doi.org/10.3141/2017-02.

Liu, Y., \& Charles, P. (2013). Spreading peak demand for urban rail transit through differential fare policy: a review of empirical evidence. In Proceedings of the Australasian Transport Research Forum 2013 (p. 1-35). Brisbane: ATRF.

McGeehan, H. (1984). Forecasting the demand for inter-urban railway travel in the Republic of Ireland. Journal of Transport Economics and Policy, 18, 275-291.

Metro de Santiago. (2016). Tarifas. Recuperado em 16 de abril de 2016, de http://www.metrosantiago.cl/guiaviajero/tarifas

Oliveira, A. V. M.; Pamplona, D. A. \& Paulo Filho, D. P. P. (2015). Estudo e previsão de demanda do transporte urbano coletivo na Região Metropolitana de São Paulo. Revista dos Transportes Públicos - ANTP, 37, 61-73.

Owen, A. D., \& Phillips, G. D. A. (1987). The characteristics of railway passenger demand. An econometric investigation. Journal of Transport Economics and Policy, 21, 231-253.

Rantzien, V. H., \& Rude, A. (2014). Peak-load pricing in public transport: a case study of Stockholm. Journal of Transport Literature, 8(1), 52-94. http://dx.doi.org/10.1590/S2238-10312014000100004.

São Paulo. Assembleia Legislativa do Estado de São Paulo - ALESP. (2015). Projeto de Lei no 1.442/2015. Autoriza o Poder Executivo a conceder redução nas tarifas dos transportes públicos do Estado em horários alternativos aos de grande fluxo. Brasília: Diário Oficial da União. Recuperado em 10 de maio de 2017, de https://www.al.sp.gov.br/propositura/?id=1284962

Suman, H. K., Bolia, N. B., \& Tiwari, G. (2017). Comparing public bus transport service attributes in Delhi and Mumbai: Policy implications for improving bus services in Delhi. Transport Policy, 56, 63-74.

http://dx.doi.org/10.1016/j.tranpol.2017.03.002.

Superintendência Executiva de Mobilidade Urbana de João Pessoa - SEMOB/JP. (2016). Dados sobre o Sistema de Transporte Coletivo Convencional. Recuperado em 16 de abril de 2016, de http://www.joaopessoa.pb.gov.br/secretarias/semob/onibus/

Transport for London. (2016). Fares. Recuperado em 16 de abril de 2016, de https://tfl.gov.uk/fares-andpayments/fares/single-fare-finder

\section{Editor: Fábio Duarte}

Recebido: Jan. 31, 2018

Aprovado: Jun. 22, 2018 